\title{
DESIGN CONCEPT FOR A NOVEL SQUID-BASED MICRODOSEMETER
}

\author{
S. Galer ${ }^{1,2, *}$, L. Hao ${ }^{1}$, J. Gallop ${ }^{1}$, H. Palmans ${ }^{1}$, K. Kirkby ${ }^{2}$ and A. Nisbet ${ }^{2,3}$ \\ ${ }^{1}$ National Physical Laboratory, Hampton Road, Teddington, Middlesex TW11 0LW, UK \\ ${ }^{2}$ Faculty of Engineering and Physical Sciences, University of Surrey, Guildford, Surrey GU2 7XH, UK \\ ${ }^{3}$ Royal Surrey County Hospital, Egerton Road, Guildford GU2 7XX, UK \\ *Corresponding author: s.galer@surrey.ac.uk
}

\begin{abstract}
The interest in proton and ion-beam therapy has increased substantially in recent years, leading the Bureau International des Poids et Mesures to recommend that a new quantity be defined to account for the biological effect of treatment modalities used in the radiotherapy. In response, we have begun work on the design of a new microbolometer based on the inductive superconducting transition-edge detector (ISTED) designed at the National Physical Laboratory. Our work aims to expand the use of the ISTED from its current use as an infra-red detector to the measurement of energy deposition of photons, hadrons and ions by incorporating a tissue-equivalent absorber. We present here the work carried out till date, including the detection of single visible photons, with an energy resolution of $0.2 \mathrm{eV}$ and a time response of a few microseconds, using a 15$\mu \mathrm{m}$ Superconducting QUantum Interference Device operating at $9 \mathrm{~K}$. We also describe thermal computational modelling carried out for a simple ISTED absorber. Finally, we discuss the work currently in progress and that to be carried out before the detector is realised.
\end{abstract}

\section{INTRODUCTION}

The number of patients being treated with ion-beam therapy, particularly with protons and carbon ions (hadrons), have increased rapidly since 1990: as the technology improves, and the cost decreases, the number of patients being treated each year is likely to increase significantly ${ }^{(1)}$. Consequently, the understanding of the biological effect of these treatment modalities becomes ever more important. Contrary to the situation for high-energy X-ray beams, the biological effect of proton and ion beams is not proportional to the absorbed dose. The Bureau International des Poids et Mesures has recently recommended the definition of a new quantity to account for the biological effect of ionising radiation used in radiotherapy ${ }^{(2)}$. The measurement of microdosimetric energy deposition distributions is part of the process to establish such a quantity.

Several methods currently exist for the measurement of microdosimetric spectra, including most significantly, tissue-equivalent proportional counters (TEPCs) $^{(3)}$, silicon-on-insulator detectors $(\mathrm{SOIs})^{(4)}$ and monolithic silicon detectors ${ }^{(5)}$. While the technology for these detectors is constantly improving and the designs are being refined, both SOI detectors and TEPCs are limited by the requirement of a tissue-equivalent conversion factor. Additionally, the TEPCs also rely on the assumption that energy deposited is directly proportional to the ionisation measured.

The use of superconducting transition edge sensors (TES) as detectors of particles or photons, read out by Superconducting QUantum Interference
Devices (SQUIDs), is now widespread (see, for example, ref. ${ }^{(6)}$ ) and rightly so as they offer many advantages over alternative detectors. These include their ability to resolve particle energy as well as to count particles due to the small superconducting energy gap of $\sim 1-2 \mathrm{meV}$ for $\mathrm{Nb}$. The fast-response times of the order of microseconds or better for micrometre-sized detectors and also the fact that superconductivity provides low noise at an easily achievable temperature imply additional advantages.

In this paper, we introduce a new type of TES, the inductive superconducting transition-edge detector (ISTED $)^{(7)}$, which has a number of potential improvements over conventional TES sensors and we propose that this design will be suitable for microdosimetry applications. In addition to a description of fabrication and the performance of a prototype device that has been tested as a single-photon detector at visible frequencies, we have begun to model the response of the ISTED to incident ion or other massive particle beams.

\section{DESIGN CONCEPT}

The design of our microbolometer is based on a new type of SQUID, which has been developed in collaboration between the National Physical Laboratory (NPL) and Surrey University ${ }^{(8)}$.

A SQUID consists of a superconducting magnetic flux-quantising ring, interrupted by one or more Josephson junctions. Traditional SQUIDs use trilayer tunnel junctions as the Josephson element. In order to reduce the size of the SQUID sensor 


\section{S. GALER ET AL.}

towards the nanoscale and to reduce the complexity of the fabrication process, we have moved towards using micro/nanobridge Josephson junctions instead. These are fabricated by focused ion-beam milling of simple thin bi-layer films consisting of high-quality sputtered $\mathrm{Nb}(\sim 150 \mathrm{~nm}$ thick $)$ with an overlay of amorphous tungsten $(\sim 60 \mathrm{~nm}$ thick), which serves both as a resistive shunt and as a sacrificial layer against ion beam poisoning of the $\mathrm{Nb}$ film. These devices have some of the lowest noise figures ever achieved for SQUIDs operated above $1 \mathrm{~K}^{(9)}$.

We propose to build a detector with a tissueequivalent absorber, likely to be amorphous carbon, with dimensions comparable to that of a cell or cell nucleus. This will be surrounded by a SQUID loop and used in radiation fields for hadrons and heavy ions. While it is possible for the detector to mimic the size of either an entire cell or a cell nucleus, the actual dimensions will be subject to the best performance predicted by computational simulations.

\section{ISTED PRINCIPLE OF OPERATION}

Traditional superconducting transition-edge sensors use a sensitive resistive component as an absorber, either current- or voltage-biased, which introduces a dissipative heat load and causes degradation of energy sensitivity through Johnson noise. In contrast, the absorber of our detector is deposited inside the loop of the readout device SQUID (Figure 1), removing the source of Johnson noise.

During operation, particle or photon absorption, or ionisation along the track length, causes the breaking of superconducting Cooper pairs. In the case of ionising radiation, the energy is usually deposited via ionisation along the track length, although a contribution is due to the ionising particle being brought to rest at the end of the track length. This results in a change in superconducting penetration depth and thus also in thin-film absorber inductance which is detected using the SQUID loop.

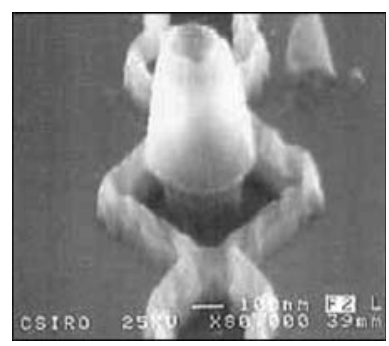

Figure 1. SEM image of a nano-SQUID containing an amorphous carbon absorber deposited on an ultra-thin $(\sim 15 \mathrm{~nm}) \mathrm{Nb}$ film deposited within the diamond-shaped SQUID loop.
The periodic response of the SQUID is altered by the change in penetration depth and this results in a change in the output voltage of the DC-currentbiased SQUID. This detection method does not require the absorber to leave the superconducting state, removing the additional sources of noise this can introduce, such as Johnson noise. The SQUID loop can vary in size from a diameter of $100 \mu \mathrm{m}$ down to $200 \mathrm{~nm}$. An example of this latter type of detector is shown in Figure 1; it uses an amorphous carbon absorber grown on a thin $\mathrm{Nb}$ film, within a SQUID loop that is grown with a thicker Nb film.

To demonstrate the efficacy of the ISTED principle, we have built a prototype device aimed at single visible photon detection. A $15-\mu \mathrm{m}$ diameter SQUID with a smaller and thinner $\mathrm{Nb}$ absorber layer inside the loop was fabricated (Figure 2a).

This was tested by shining a fibre-coupled ultraweak 633-nm laser beam onto the absorber in order to determine the energy sensitivity of the detector, as well as the time resolution. Figure 3 shows histogram plots of the number of detected pulses against pulse amplitude. Figure 3a (with the laser on) shows a peak in the distribution at an amplitude of around 0.05 , which corresponds to single laser photon detection. The peak width indicates that the energy resolution of this device is around $0.2 \mathrm{eV}$, even at the relatively high operating temperature of 9.067 K. Figure $3 \mathrm{~b}$ shows the same plot with the laser turned off. Note the sharp rise in counts at lowpulse amplitudes. We believe this is due to blackbody infrared radiation being conducted down the fibre from its warm $(300 \mathrm{~K})$ end. The inset shows a trace of SQUID output amplitude against time, indicating single photon absorption pulses and the response time of the detector, which is of the order of a few microseconds.

\section{THERMAL MODELLING FOR MICRODOSIMETRY}

Typical energy depositions from ionising radiation are obviously much higher than from IR photons. For example, electrons liberated by high-energy $\mathrm{X}$ rays lose, on average, $\sim 200 \mathrm{eV} \mu \mathrm{m}^{-1}$, while protons and carbon ions of $10 \mathrm{MeV}$ lose, on average, $\sim 5000$ and $80000 \mathrm{eV} \mathrm{\mu m}^{-1}$, respectively. We have begun to model the thermal behaviour of the ISTED absorber in response to the absorption of the kinetic energy of an impacting massive particle such as an ion. Here, we wish to simulate tissue-equivalent material as the upper layer of the absorber, coupled thermally to a thin, scarcely perturbing, layer of superconductor below. The thermal diffusivity of the combined system is the key physical parameter that determines the thermal response. In view of the complexity of the geometry, we have been using a finite element package (COMSOL) to model both time and spatial 


\section{DESIGN CONCEPT FOR SQUID MICRODOSEMETER}
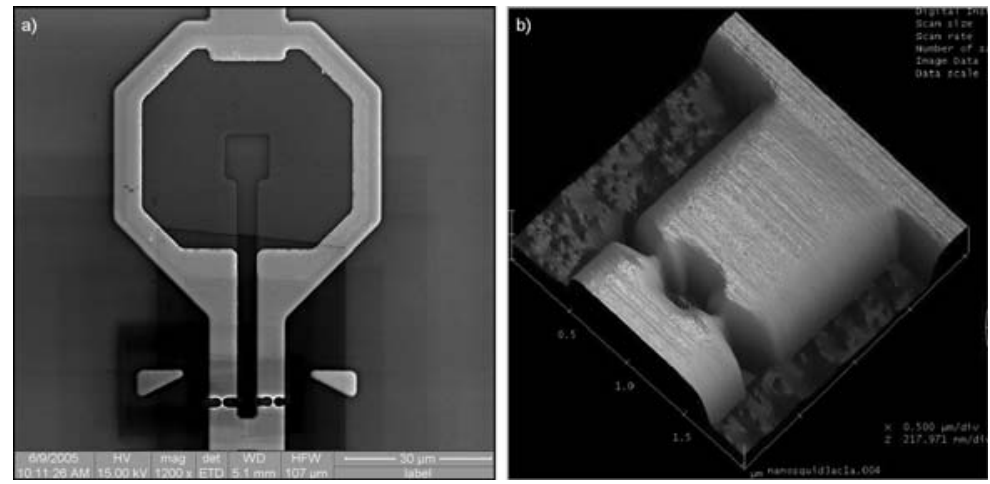

Figure 2. (a) A 50- $\mu \mathrm{m}$ SQUID with $\mathrm{Nb}$ absorber within the square ring. (b) An AFM image of a nano-SQUID, highlighting the size of the microbridge junctions that are typically $60-80 \mathrm{~nm}$ in width and length.
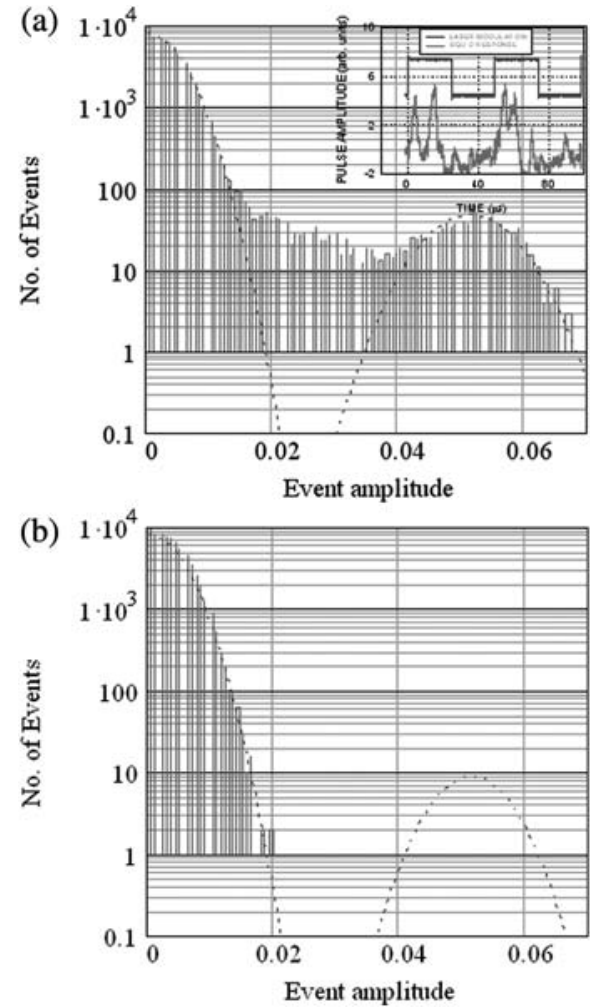

Figure 3. Histograms of number of SQUID output events versus event amplitude: (a) with weakly coupled 633-nm laser illuminating absorber. Inset shows single photon pulses versus time. (b) Same plot with laser off. Note the logarithmic vertical axis.

variation of temperature following an absorption event. Figure 4 shows a snapshot of the temperature distribution of an absorber element some $0.3 \mu \mathrm{s}$ after total absorption of a $1-\mathrm{MeV}$ incident particle.

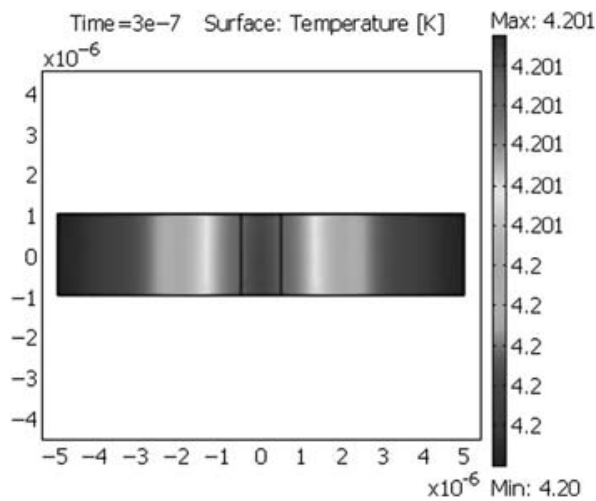

Figure 4. Plot of temperature distribution $0.3 \mu$ s after the absorption of a $1-\mathrm{MeV}$ particle. Note that the thermal hot spot has spread by around $2 \mu \mathrm{m}$ in this time. The temperature rise produced $(\sim 1 \mathrm{mK})$ is more than enough for the detection sensitivity of the ISTED.

\section{PROPOSED SETUP}

Figure 5 shows a picture of a compact liquid helium cryostat (height $\sim 60 \mathrm{~cm}$ ) similar to the one that will be used for this work, along with a schematic of the intended experimental setup of the microbolometer during irradiation. The detector is fixed to a thermally conducting block that is partially isolated from the helium bath by a thermal resistance. The block can then be heated above the $4.2 \mathrm{~K}$ of the bath in order to maintain a temperature close to $T_{\mathrm{c}}$, the superconducting transition temperature.

\section{CONCLUSIONS}

Work carried out to date indicates that the proposed ISTED-type detector is well suited to the measurement of microdosimetric spectra, providing a suitable absorber can be designed. 
(a)

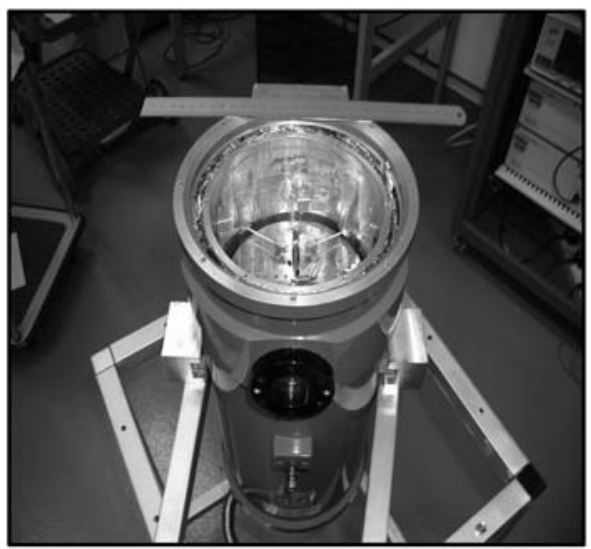

(b)

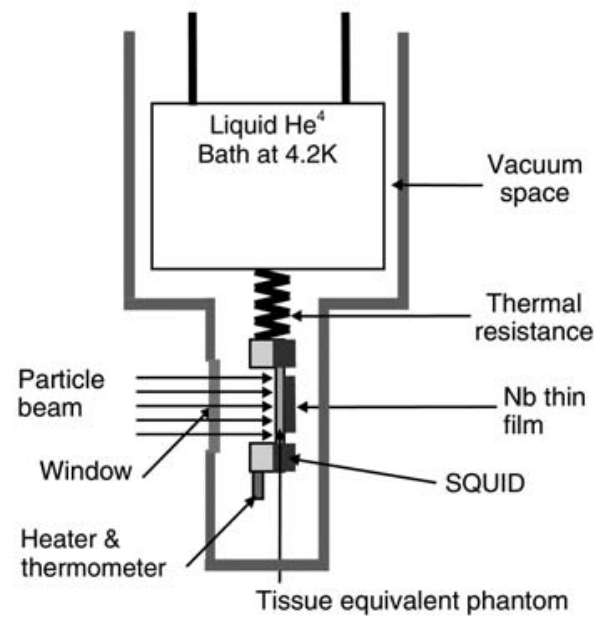

Figure 5. (a) Photograph of the liquid Helium-4 cryostat; (b) schematic of how the microbolometer will be set up during operation.

The first detectors produced are intended as proof-of-principle for this new technology applied to the detection of charged particles and ions. A major challenge that must be overcome in creating a successful tissue-equivalent device is that of the scatter contributions from areas of the device other than the absorber. It is our intention to investigate means of minimising these energy contributions after the technology has been demonstrated successfully. Solutions to be investigated include reducing the thickness of the substrate, deriving a correction factor from Monte-Carlo simulations and considering alternative layouts in order to minimise scatter.

The visible photon work carried out suggests that the detection of single particles should be attainable and that detection times should be of the order of a few microseconds, depending on the thermal coupling of the absorber. Initial thermal modelling indicates that the response time should not be significantly affected by the thermal recovery although, again, this depends upon the thermal coupling achieved. It is hoped that we can obtain detector sensitivity of $\sim 0.2 \mathrm{eV}$, although this may be challenging for energetic particles with energies of $\sim 0.1 \mathrm{MeV}$ or greater.

As the detector will be subjected to proton and ion beams, it is likely to eventually suffer from radiation damage or poisoning. While some contamination of the SQUID is likely, by minimising unnecessary contamination, we believe that it will not become significant for our initial proof of principle experiment.

\section{FURTHER WORK}

Current work on this project revolves around the microdosimetric computational modelling of the absorber that will be deposited within the SQUID ring. This work strives to optimise the shape and dimensions of the absorber as well as investigating several candidate materials to be used.

The computational modelling can be broken into two separate sections. Firstly, radiation modelling is being carried out using both the Geant 4 and FLUKA radiation Monte-Carlo codes. This work is carried out in order to investigate how the shape and dimensions of the absorber affect the energy deposition within the device. Secondly, the thermal modelling previously carried out is to be continued in order to simulate the thermal transport of the candidate materials and prospective designs to better understand how the device should respond.

Following the completion of this work, it is our aim that several prototypes will be designed and created that can then be tested and compared with theoretical predictions. From here further modifications and improvements can be made as required, along with investigations into optimum detector size, and radiation harness, before a completed working device can be produced.

\section{ACKNOWLEDGEMENTS}

The authors thank John Macfarlane for his assistance and expertise. This paper is subject to Crown Copyright and is reproduced by permission of the Controller of HMSO.

\section{FUNDING}

This work is funded through the NPL-Surrey Strategic Partnership.

\section{REFERENCES}

1. Sisterson, J. Ion beam therapy in 2004. Nucl. Instrum. Methods Phys. Res. B 241, 713-716 (2005). 


\section{DESIGN CONCEPT FOR SQUID MICRODOSEMETER}

2. BIPM-CCRI. Report of the 19th Meeting, Bureau International des Poids et Mesures. Consultative Committee for Ionizing Radiation Report, BIPM. Published online at http://www.bipm.org/utils/common/ pdf/CCRI19.pdf (2005).

3. Rossi, H. H. and Zaider, M. Elements of microdosimetry. Med. Phys. 18, 1085-1092 (1991).

4. Wroe, A., Rosenfeld, A., Reinhard, M., Pisacane, V., Zeigler, J., Nelson, M., Cucinotta, F., Zaider, M. and Dicello, J. Solid state microdosimetry with heavy ions for space applications. IEEE Trans. Nucl. Sci. 54(6), 2264-2271 (2007).

5. Agosteo, S., Fallica, P. G., Fazzi, A., Introini, M. V., Pola, A. and Valvo, G. A pixilated silicon telescope for solid state microdosimetry. Radiat. Meas. 43(2-6), 585-589 (2008).

6. Woodcraft, A. L., Ade, P. A., Bintley, D., House, J. S., Hunt, C. L., Sudiwala, R. V., Doriese, W. B., Duncan,
W. D., Hilton, G. C. and Irwin, K. D. et al. Electrical and optical measurements on the first SCUBA-2 prototype 1280 pixel submillimeter superconducting bolometer array. Rev. Sci. Instrum. 78, 024502 (2007).

7. Hao, L., Gallop, J. C., Gardiner, C., Josephs-Franks, P., Macfarlane, J. C., Lam, S. K. H. and Foley, C. Inductive superconducting transition-edge detector for single-photon and macro-molecule detection. Supercond. Sci. Technol. 16, 1479-1482 (2003).

8. Hao, L., Macfarlane, J. C., Lam, S. K. H., Foley, C. P., Josephs-Franks, P. and Gallop, J. C. Inductive sensor based on nano-scale SQUIDs. IEEE Trans. Appl. Supercond. 15(2), 514-517 (2005).

9. Hao, L., Macfarlane, J. C., Gallop, J. C., Cox, D., Beyer, J., Drung, D. and Schurig, T. Measurement and noise performance of nano-superconducting quantum interference devices fabricated by focused-ion-beam. Appl. Phys. Lett. 92, 192507 (2008). 